Auch wenn das BVerfG sich zu diesen Zulagen aus Fraktionsmitteln nicht geäußert hat, halten sie die Autoren für eindeutig verfassungswidrig. Zuzustimmen ist ihnen zumindest darin, dass solche Zulagen deutlich transparenter als bisher gemacht werden sollten. Darüber hinaus kann man aber durchaus die Frage stellen, warum die Fraktionen besondere Leistungen ihrer Mitglieder, die sie im Zweifel auch extern „einkaufen“ beziehungsweise von Mitarbeitern erledigen lassen könnten (Beispiele: Geschäftsführung, Justiziariat), nicht vergüten dürfen. Vielleicht wäre es sogar sinnvoller, sämtliche Zulagen, also auch für den Fraktionsvorsitz, ausschließlich aus der Fraktionskasse zu zahlen, weil es Sache der Fraktionsautonomie ist, ob und welche Funktionen ihre Mitglieder für notwendig erachten (zum Beispiel einen oder mehrere Fraktionsvorsitzende) und ob und in welcher Höhe sie dafür zahlen wollen. Im Gegenzug wäre ein Höchstmaß an Transparenz für solche Vergütungen erforderlich, verbunden mit einer Steuerung und Begrenzung der Fraktionszuschüsse zum Beispiel - wie bei der Parteienfinanzierung - mittels einer Indexierung.

Dass steuerfreie Aufwandspauschalen von Verfassungs wegen Bestandteil der Diäten sein können, ist mittlerweile gefestigte Rechtsprechung - sofern sie sich am tatsächlichen Aufwand orientieren. Von Armin und Drysch zeigen allerdings so eklatante Unterschiede bei den entsprechenden Regelungen in Bund und Ländern auf, dass ihre Frage berechtigt ist, ob wirklich alle Regelungen diesem Maßstab gerecht werden. Einige Parlamente verzichten mittlerweile auf steuerfreie Aufwandspauschalen.

Insgesamt haben von Arnim und Drysch eine Kommentierung vorgelegt, die durch ihren Faktenreichtum und insbesondere durch die Einbeziehung der Länderregelungen besticht - auch wenn das die Gefahr mit sich bringt, dass die Reichweite des Art. 48 GG auf die Länder gelegentlich überdehnt wird. Bei dem als „Diätenkritiker“ bekannten Autor von Arnim wundert es nicht, dass Kritik an bestehenden Regelungen häufig und deutlich geäußert wird. Sie bleibt aber im Rahmen dieses Kommentars stets sachlich; und auch wenn die Argumentation gelegentlich etwas oberflächlich ist und nicht in jedem Fall überzeugt, lohnt sich die Auseinandersetzung mit ihr. Es bleibt zu hoffen, dass die nächste Neubearbeitung nicht gar so lange auf sich warten lässt.

Florian Edinger

\title{
Schuldenbremse im Grundgesetz: verteidigt und fortentwickelt
}

Ryczewski, Christoph: Die Schuldenbremse im Grundgesetz. Untersuchung zur nachhaltigen Begrenzung der Staatsverschuldung unter polit-ökonomischen und bundesstaatlichen Gesichtspunkten (Schriften zum Öffentlichen Recht, Band 1182), Duncker \& Humblot, Berlin 2011, 261 Seiten, $€ 58,-$.

Christoph Ryczewskis an der Humboldt Universität entstandene Dissertation greift ein tagespolitisch brisantes Thema auf. Sie untersucht die Ausgestaltung und die Defizite der seit 2009 durch die Föderalismusreform II im Grundgesetz verankerten Schuldenbremse. Die ersten circa 140 Seiten der Studie sind Bekanntem gewidmet, nämlich der Vorgeschichte dieser Reform, also den bisherigen Verschuldungsregeln auf Bundes-, Landes- und Europaebene. Deren ausführliche Betrachtung soll belegen, dass 2009 tatsächlich Reformbedarf 
bestand, was kaum zu bestreiten ist. Die Literaturbasis dieser Betrachtungen, wie auch der gesamten Arbeit, schließt zwar Rational Choice-Ansätze aus der Volkswirtschaftslehre mit ein (der Autor nennt diese polit-ökonomisch), vernachlässigt aber andere, insbesondere sozialwissenschaftliche Zugänge zum Gegenstand der Staatsverschuldung. Dies ist angesichts virtueller Disziplinengrenzen nicht unüblich und deshalb dem Autor nicht vorzuhalten; allerdings wäre gerade bei seinen Erörterungen zur demokratischen Legitimation der Schuldenbremse und zur politischen Rolle der Länder im Bundesstaat die Auseinandersetzung mit der sozialwissenschaftlichen Literatur hilfreich gewesen.

Was dieses Buch besonders lesbar macht, ist nicht nur die gedankliche Klarheit der Argumentation, sondern auch das direkte Zugehen des Autors auf die Kernprobleme seines Gegenstandes. Da ist zum einen der Vorwurf, die Schuldenbremse verstoße gegen das Bundesstaatsprinzip. Dem tritt der Autor im Hinblick auf die Haushaltsautonomie der Länder entgegen, ,solange anderweitig für eine aufgabenadäquate Finanzausstattung gesorgt ist“ (S. 162) - ob dies aber so ist, lässt sich nur empirisch beantworten und deshalb weniger eindeutig, als dies Ryczewski tut.

Des Weiteren wurde kritisiert, die Schuldenbremse verstoße gegen das Demokratieprinzip, weil die Landesparlamente hinsichtlich ihres „Königsrechts“ Budgetaufstellung fremdbestimmt wurden. Auch diesen Vorwurf weist der Autor zurück und begründet dies mit einem hinreichend großen Legitimationsniveau der Bundesratsentscheidung (S. 167). Dies verwundert nicht nur, weil der Bundesrat ein Bundesorgan ist, sondern vor allem, weil einer Legitimation der Schuldenbremse durch die Landesparlamente durch diese selbst so wenig Bedeutung beigemessen wird. Dies ist, wie das Zustandekommen von balanced budget-Regeln auf Gliedstaatenebene in anderen Bundesstaaten zeigt, aus guten Gründen im Föderalismus eine unübliche Argumentation. Den Landtagen wird nun beispielsweise zugemutet, der Schuldenbremse folgend Sparpolitik zu betreiben, ohne dass die Abgeordneten sich für oder gegen die Schuldenbremse gegenüber ihren Wählern geäußert hätten.

Strittig ist drittens die Frage, ob das Grundgesetz mit den Detailregelungen des Artikels 143d, die sogar Festbeträge für Konsolidierungshilfen enthalten, „deformiert“ wurde. Dies ist nicht nur ein „verfassungsästhetisches“ Problem. Ryczewski plädiert für Detailliertheit, weil er argumentiert, Begleitgesetze unterlägen der Gefahr der Änderung und damit der Aushöhlung der Schuldenbremse, denn sie binden den Gesetzgeber selbst nicht. Auch das Grundgesetz kann natürlich geändert werden. Es ist zu vermuten, dass der Gesetzgeber vor allem an die höhere Hürde bei Verfassungsänderungen im Sinne der Selbstbindung gedacht hat.

Bei der Bewertung der Föderalismusreform II kommt der Autor zu zustimmenden und kritischen Urteilen. Letztere entwickelt er weiter zu einem eigenen Vorschlag für eine Reformulierung der entsprechenden Artikel des Grundgesetzes. Es fällt auf, dass Ryczewski zwei Reformelemente positiv sieht, die beispielsweise die OECD deutlich kritisiert hat. Zum einen ist dies die konjunkturelle Verschuldungskomponente, die er für „eindeutig bestimmbar“ (S. 179) hält. Laut OECD „ist allerdings schwierig, ein verlässliches Maß für den Konjunkturzyklus zu ermitteln, da sich das Produktionspotenzial nicht beobachten lässt und jede Messung der Lücke zwischen effektiver und potenzieller Produktion somit in hohem Maße ungewiss ist und häufig Gegenstand erheblicher Revisionen im Zeitverlauf wird" . Zum anderen klammert er aus systematischen Gründen den Stabilitätsrat aus sei-

1 OECD, Wirtschaftsberichte Deutschland, Paris 2010, S. 69 f. 
nen Betrachtungen aus, weil er nicht „schadensvorbeugend“ wirke, sondern „schadensbegrenzend“ (S. 189). Dies unterschätzt die Monitoring-Aufgabe des Stabilitätsrats und umgeht das Problem, das die OECD nennt: „In seiner derzeitigen Zusammensetzung gehören dem Rat jedoch die Finanzminister von Bund und Ländern an, so dass die Bundesregierung und die Länderregierungen sich im Wesentlichen selbst kontrollieren. Das könnte dazu führen, dass der Rat zu lax auf Haushaltsüberschreitungen oder Haushaltskosmetik reagiert." 2

Sehr überzeugend sind die Änderungsvorschläge, die der Autor entwickelt. Er plädiert für einen Wegfall der strukturellen Verschuldungsmöglichkeit des Bundes, weil er realistischerweise erwartet, dass diese immer vollständig genutzt werden wird, womit sich die Gesamtverschuldung permanent erhöht. Beim Verstoß gegen Verschuldungsregeln schlägt er einen Zuschlag zur Einkommensteuer vor, um die durch unzulässige Ausgaben entstandene Lücke zu stopfen. Hier soll der Wähler auf Regierungshandeln aufmerksam gemacht werden und an der Wahlurne Konsequenzen ziehen. Ryczewski erhofft sich auch einen Mentalitätswechsel in der Politik dahingehend, dass es nicht um „Staatsausgaben“ geht, sondern um das Geld des Steuerzahlers beziehungsweise Wählers. Hinsichtlich der Tilgungsregeln in den Ausnahmefällen, in denen eine Abweichung von den neuen Haushaltsregeln erlaubt ist (Notsituationen) wünscht sich der Autor präzisere Festlegungen, etwa eine Regelfrist von fünf Jahren. Im Augenblick ist in Artikel 115 (2) GG nur von einem „angemessenen Zeitraum" die Rede. Weiterhin diskutiert Ryczewski Vorschläge, den Kreis möglicher Klageberechtigter beim Bundesverfassungsgericht zu erweitern. Er präferiert ein Antragsrecht für die Parlamentsfraktionen gegenüber einem solchen für den Bundesrechnungshof, der zwar fachlich kompetent aber demokratisch schwächer legitimiert sei. In der Summe ist Christoph Ryczewski zu bescheinigen, dass ihm ein sehr kluger Beitrag zum Thema „Schuldenbremse" gelungen ist.

Roland Sturm

2 Ebenda, S. 73.

\section{Deutschland und Europa: weiter Blick zu Ehren von William Paterson}

Bulmer, Simon, Charlie Jeffery und Stephen Padgett (Hrsg.): Rethinking Germany and Europe. Democracy and Diplomacy in a Semi-Sovereign State, Palgrave Macmillan, Houndsmills 2010, 243 Seiten, $€ 71,99$.

Die britische Politikwissenschaft hat in den letzten Jahren und Jahrzehnten die Einbindung der Bundesrepublik in die europäischen Strukturen intensiv begleitet. Zu deren exponiertesten Autoren gehört zweifellos William Paterson, um dessen Wirken sich der angezeigte Band dreht. Das von Simon Bulmer, Charlie Jeffery und Stephen Padgett - drei weiteren Exponenten der britischen Deutschlandforschung - edierte Werk stellt eine etwas ungewöhnliche Festschrift dar, wie die Herausgeber in ihrer Einleitung selbst anmerken (S. 2). Viele der hier versammelten Texte rekurrieren direkt auf Paterson, allerdings häufig eher am Ran- 